\title{
AUTORADIOGRAPHIC ANALYSIS OF ODONTOBLAST REPLACEMENT FOLLOWING PULP EXPOSURE IN PRIMATE TEETH
}

\author{
M. FitzGerald, ${ }^{1 *}$ D. J. Chiego J $\mathbf{R}^{2}$ and D. R. Heys ${ }^{1}$ \\ 'Department of Cariology and General Dentistry and ${ }^{2}$ Department of Biologic and Materials Sciences, \\ The University of Michigan, School of Dentistry, Ann Arbor, MI 48109-1078, U.S.A.
}

(Accepted 26 April 1990)

\begin{abstract}
Summary-Cell migration and replication associated with odontoblast replacement occurring soon after pulp exposure in primate teeth were studied. Class 5 cavity preparations resulting in pulp exposures were restored with a calcium hydroxide-containing capping agent and amalgam. Eighty-four and $96 \mathrm{~h}$ after this the animals were injected with $0.5 \mu \mathrm{Ci} / \mathrm{g}$ body wt tritiated thymidine (sp. act. $6.7 \mathrm{Ci} / \mathrm{mM}$ ). Teeth were extracted 6, 8, 10 and 12 days after treatment. The number of labelled cells as well as the number of grains per labelled cell were counted for odontoblast-like, fibroblast-like and perivascular cells in three $60 \times 260 \mu \mathrm{m}$ zones. These zones represented the odontoblast and cell-free (zone 1), cell-rich (zone 2) and deep pulp (zone 3) areas of normal pulp tissue. Ten sections centred around the mid-point of the exposure were counted for each tooth. Matrix formation and labelled odontoblast-like cells were observed at the interface between the capping agent and the pulp as early as day 8. Other significant findings were: (1) an increase in labelled odontoblast-like cells in zone 1 over time, suggesting a continual influx of differentiating cells; (2) an increase in labelled cells in zone 1 over time with a concurrent decrease in zone 3 , suggesting that the influx of cells in zone 1 was from the deeper pulp; and (3) differences in grain counts between zones, treatment times and cell types, indicating that at least two DNA replications had occurred between initial treatment and final odontoblast-like cell differentiation.
\end{abstract}

Key words: autoradiography, pulp, repair, regeneration odontoblasts.

\section{INTRODUCTION}

Although the scquence of healing after direct capping of pulp exposures have been well described, the patterns of pulpal cell migration and replication associated with these sequences, cspecially odontoblast replacement, are not well understood. Several sources of replacement odontoblasts have been suggested: these iriclude (1) $\mathbf{G}_{2}$-blocked, predetermined cells that require no DNA replication for differentiation into functioning odontoblasts after induction; and (2) cells in the pulp proper that replicate their DNA after induction and before differentiating into functioning odontoblasts.

The existence of a $\mathrm{G}_{2}$-blocked, predetermined population of odontoblast replacement cells was suggested by Stanley (1962), Takuma and Nagai (1971), and Slavkin (1974), because of the similar morphology of cells in the cell-rich zone and the adjacent functioning, mature odontoblasts. Findings by Cotton (1968) and Torneck and Wagner (1980) seem to support this theory: they found no $\left[{ }^{3} \mathrm{H}\right]$-thymidine-labslled odontoblast-like cells in rat molar pulps after tooth grinding without pulp exposure, which suggests that odontoblasts destroyed by the grinding were replaced by predetermined cells that did not replicate their DNA in response to the injury. If these $G_{2}$-blocked, predetermined cells do exist, they would most likely reside in the cell-rich

*To whom correspondence should be addressed. zone of the pulp (Ruch, 1984; Veis, Tsay and Kanwar, 1984).

Others have proposed that replacement odontoblasts are derived from either undifferentiated mesenchymal cells in the pulp proper that are induced to differentiate into odontoblasts or from differentiated pulpal cells that are induced to dedifferentiate into undifferentiated cells and then redifferentiate into odontoblasts (Fitzgerald, 1979; Yamamura et al., 1980; Yamamura, 1985; Tziafas and Kolokuris, 1990). This process would require recruitment and induction of a cell population(s) within the pulp proper after pulpal trauma, one or more DNA replication cycles and finally differentiation into functioning odontoblasts. Studies with $\left[{ }^{3} \mathrm{H}\right]$-thymidine in wounded, non-exposed pulps (Sveen and Hawes, 1968 ) as well as in pulps wounded by exposure (Feit, Metelova and Sindelka, 1970; Yamamura et al., 1980; Yamamura, 1985) have demonstrated that DNA replication can occur during the replacement of odontoblasts lost due to trauma. The replacement cell population in this type of pulp healing could be located anywhere within the coronal pulp, as suggested by Fitzgerald (1979), Yamamura et al. (1980), Yamamura (1985) and Tziafas and Kolokuris (1990). The cell types involved could be from fibroblastic (Fitzgerald, 1979; Yamamura et al., 1980), vascularrelated (Yamamura et al., 1980), and undifferentiated mesenchymal (Yamamura et al., 1980) lines.

Thus, knowledge of cell replacement and replication patterns after pulpal wounding is equivocal. It 
may be that both mechanisms, $\mathrm{G}_{2}$-blocked cells and DNA replication and differentiation of undifferentiated or dedifferentiated pulpal cells, are viable for odontoblast replacement. Further investigation of the patterns of DNA replication and cell migration after pulpal wounding and treatment would help to clarify these issues. Thus, our purpose now was to identify early cell migration and replication patterns associated with odontoblast replacement after pulp exposure in primate teeth.

\section{MATERIALS AND METHODS}

Five adult rhesus monkeys (Macaca mulata) were used to provide 8 teeth for each of four time periods $-6,8,10$ and 12 days. Teeth assigned to the various treatment groups were distributed between the animals so that each time period was represented in two animals. After sedation with $10 \mathrm{mg} / \mathrm{kg}$ body wt ketamine hydrochloride (Parke-Davis and Co., Detroit, MI) intramuscularly and sodium pentabarbitol intravenously, titrated to effect, Class 5 buccal cavity preparations resulting in pulp exposure and destruction of the underlying odontoblast, cell-free, cell-rich and deeper pulp tissue were cut with an ultraspeed handpiece and carbide burs with an air/water spray. Haemostasis was achieved with sterile cotton pellets, the exposed pulps and surrounding cut dentine were covered with a thin layer of a hard-setting calcium hydroxide (Life, Sybron/ Kerr, Romulus, MI) and the preparations restored with amalgam.

Eighty-four and $96 \mathrm{~h}$ after capping, each monkey was injected with $0.5 \mu \mathrm{Ci} / \mathrm{g}$ body wt of $\left[{ }^{3} \mathrm{H}\right]$-thymidine (sp. act. $6.7 \mathrm{Ci} / \mathrm{mM}$ ). These times were chosen because they were centred around the time of peak $\left[{ }^{3} \mathrm{H}\right]$-thymidine labelling activity observed by Fitzgerald (1979) in monkey pulps after similar treatment of pulp exposures. The intent of this labelling sequence was to maximize DNA labelling of pulpal cells.

At the conclusion of the treatments, the animals were sedated as described above, the appropriate teeth extracted from the alveolus, the apical one third of the root removed and the extracted teeth placed in phosphate buffered formalin-paraformaldehyde fixative for $24 \mathrm{~h}$. After demineralization in $0.5 \mathrm{M}$ EDTA and dehydration in a graded series of alcohols, the teeth were embedded in either a methylmethacrylate embedding medium (JB-4 Embedding Kit, Polysciences Inc., Warrington, PA) or paraffin. The teeth were then serially sectioned at $4 \mu \mathrm{m}$ through the exposures, dipped in Kodak NTB-2 photographic emulsion, exposed at $4^{\circ} \mathrm{C}$ for 15 days, developed, fixed and stained with toluidine blue for the methylmethacrylate-embedded teeth and haematoxylin and eosin for the paraffin-embedded teeth.

To aid in standardizing the labelled cell counting and to relate those counts to normal pulpal architecture, the pulp tissue against the capping agent at the exposure site was divided into three equal-sized $260 \times 60 \mu \mathrm{m}$ zones centred inciso-cervically in the exposure (Text Fig. 1). Zone 1 corresponded to the average thickness of the combined odontoblast and cell-free zones of sound, undisturbed pulp tissue; zone 2 corresponded to the average thickness of the cellrich zone and zone 3 was directly against zone 2 , representing the deeper pulp tissue. Three similar control zones were counted on the untreated lingual wall opposite the exposure.

Labelled perivascular, fibroblast-like and odontoblast-like cells were counted in each of the zones in ten, $16 \mu \mathrm{m}$-spaced sections that were equally centred around the mesial-distal mid-point of the exposure. The number of grains associated with the nucleus of each labelled cell, corrected for background labelling, was also recorded but not statistically tested. The autoradiographic grains were distinguished from

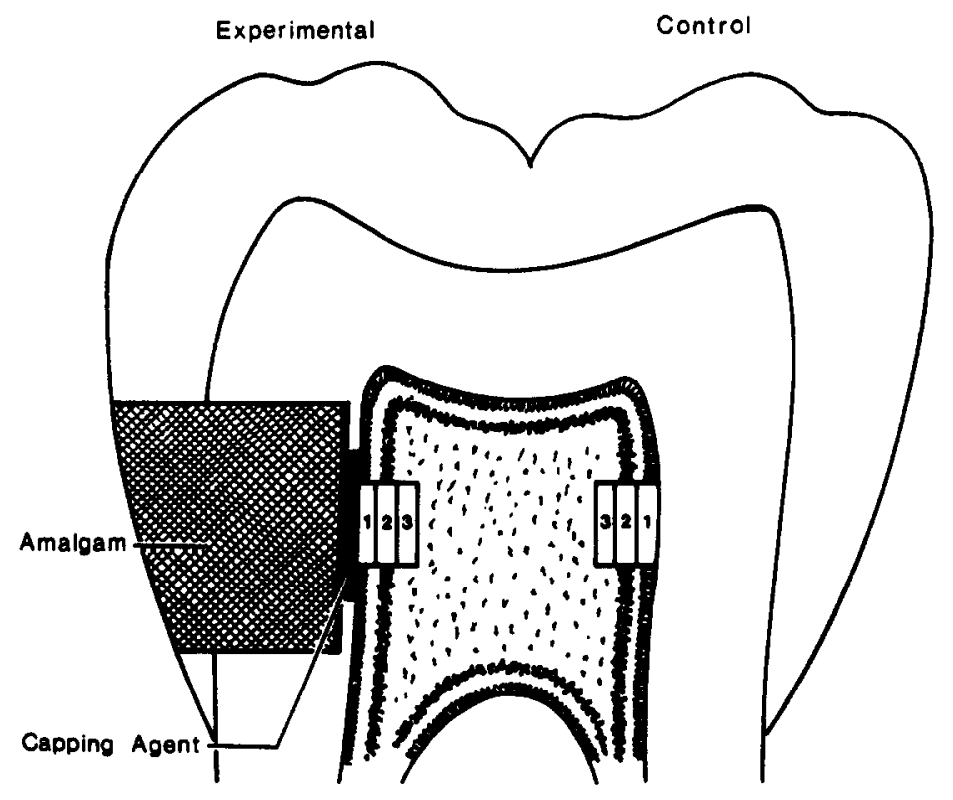

Fig. 1. Diagram showing the location of zones, 1,2 and 3 for experimental and control areas. 
similar appearing structures that originated from the capping agent by their different plane of focus and bifringence under phase-contrast microscopy, determined by the depth of field of the objective used. The following criteria were used in counting:

Labelled cells: Calls with 5 or more grains over the nucleus.

Perivasculatr cells: Flattened, elongated cells in direct association or connection with the outer surface of a vessel wall and a large centrally located nucleus.

Fibroblast-like calls: Stellate to elongated cells not associated with a vessel wall or predentinelike matrix.

Odontoblast-like cells: Cuboidal to columnar cells in zone 1 with a polarized nucleus, a cytoplasm: nucleus ratio greater than $1: 1$ and associated with a predentine-like matrix.

Only teeth with exposures large enough to permit proper positioning of the $260 \mu \mathrm{m}$ tall zone 1 in all ten, $16 \mu \mathrm{m}$-spaced sections and with enough pulpal depth at the exposure site to prevent an overlap of zone 3 of both the experimental and control areas within each tooth were used. This also ensured exposure sites large enough to have involved destruction of the original pulpal tissue in zones 1-3 at the time of treatment. Counts of all teeth in the same treatment group within one animal were combined and expressed as numbers of labelled cells per section per animal and number of grains per cell per animal.

The resultant labelled cell counts were analysed by simple regression analysis of the changes in labelled odontoblast-like cells, and repeated measures analysis of variance tests on the data for the fibroblast-like and perivascular cells at the 0.05 level of significance (Winer, 1962). Because odontoblast-like cells, by definition, could only be found in zone 1, these had to be excluded from the repeated measures analysis of variance.

\section{RESULTS}

Pulpal tissue associated with the exposure was generally free of dentine chips and other debris from cavity preparation (Plate Fig. 2). At the time of labelling, the trauma from the exposure persisted, including destruction of the odontoblast, cell-free, cell-rich and part of the deeper pulpal tissue at the exposure site (Plate Fig. 2). The tissue response at Day 6 was characterized by direct apposition of pulp tissue against the capping agent, with fibroblast-like cells aligned along the interface between the two (Plate Fig. 3). Scattered labelled fibroblast-like and perivascular cells could be seen along this interface. Cellular reorganization and matrix formation occurred at the interface, with evidence of matrix formation and odontoblast-like cells as early as day 8 (Plate Fig. 4). At this and later times, both labelled and unlabelled odontoblast-like cells (Plate Fig. 4 and $5)$, some with processes extending into the adjacent matrix (Plate Fig. 5), were seen in zone 1 at the interface. The distribution of labelled odontoblastlike cells appeared to be random and not a function of their location along the interface (Plate Figs 4 and 5).
The autoradiographic findings can be seen in Text Figs 7-12. Because of the constraints imposed by adequate exposure size and pulpal depth, the final number of teeth per treatment time was: 4 for days 6 and 8; 6 for day 10; and 5 for day 12 . One animal in the day 6 group was eliminated from the study because of medical complications unrelated to the experimental procedures, leaving only one animal in that group. The remaining times were each represented by 2 animals. All control values were less than or equal to 0.1 labelled cells per section.

The number of labelled odontoblast-like, fibroblast-like and perivascular cells per section over the 4 time periods in zone 1 is shown in Text Fig. 7 . From day 6 to day 12 there was a statistically significant increase in the number of labelled odontoblast-like cells per section in zone $1(p \leqslant 0.05)$. A significant difference between cell types $(p \leqslant 0.01)$ was observed, with labelled fibroblast-like cell counts being greater than labelled odontoblast-like and perivascular cell counts. There were no significant differences between the number of labelled odontoblast-like and perivascular cells in zone 1 over the times tested. Significant differences were found in the total number of labelled cells in zone 1 over time, with total numbers of labelled cells at day 6 being less than at days 8,10 and 12 , the total number of labelled cells at day 8 being less than at day 12 and the total number of labeled cells at day 10 being less than at day 12 $(p \leq 0.05)$. Finally, there was no statistically significant interaction between cell types (odontoblastlike, fibroblast-like and perivascular cells) and time in zone 1.

The number of labelled, fibroblast-like and perivascular cells in the three zones across the four time periods can be seen in Text Figs. 7 and 8 . There was an interaction between time and zone in the combined number of labelled, fibroblast-like plus perivascular cells, with zone 1 going from an initial low count to a high count and zone 3 going from a high count to a low count (Text Fig. $8, p \leqslant 0.05$ ). Also, at day 12 there was a difference in the total number of labelled cells across the three zones, with zone 1 being the highest, zone 2 intermediate and zone 3 lowest (Text Fig. $8, p \leqslant 0.05$ ). The relationships between zones and cell types were: a difference in the number of labelled cells across zones (zones 1 and $2>$ zone $3 ; p \leqslant 0.05$ ); a difference in cell types in zones 1 and 2 (fibroblast-like > perivascular; $p \leqslant 0.05$ ); but no difference in cell types in zone 3 (Text Fig. 9).

The grain counts can be found in Text Figs 10-12. In zone 1 counts for the odontoblast-like cells were consistent throughout the study (Text Fig. 10). Counts for fibroblast-like cells at day 6 in zone 1 were approximately twice those for odontoblast-like cells at later times but steadily fell throughout the study until they were approximately equal to those for the odontoblast-like cells at day 12 (Text Fig. 10) This trend of initially high counts steadily decreasing over time was repeated in the fibroblast-like cell population in zones 2 and 3 (Text Figs. 11 and 12). Grain counts for the perivascular cell population were variable and generally higher than for the fibroblastlike cells (Text Figs. 10-12). The counts for fibroblast-like cells at each time period were also slightly 
higher for zone 2 than zone 1 and for zone 3 than zone 2 (Figs 10-12).

\section{DISCUSSION}

The pulpal responses to our capping procedures were similar to those described by Fitzgerald (1979). The exposures, created with the carbide burs, resulted in the destruction of the odontoblast, cell-free and cell-rich zones as well as variable amounts of subjacent pulpal tissue at the exposure site (Plate Fig. 2). After capping, this destruction then underwent various stages of resolution leading to organization of fibroblast-like cells along the interface between pulp tissue and the capping agent (Plate Fig. 3) and ending with the appearance of new cells along that interface which appeared to be functioning as odontoblasts (i.e. oriented perpendicular to the exposure site with their apical ends associated with a dentine-like matrix) (Plate Figs 4 and 5). If adjacent, undisturbed mature odontoblasts are irreversible postmitotic cells, as suggested by Feit, Metelova and Sindelka (1970) and Slavkin, Zeichner-David and Siddiqui (1981), then these replacement odontoblast-like cells were derived from the surrounding pulp proper.

The presence of labelled odontoblast-like cells along the interface (Plate Figs 4 and 5) was of particular interest because it indicates that these replacement cells had replicated their DNA before differentiating into functioning odontoblasts at the interface. As all of the original odontoblasts at the exposure site had been earlier destroyed (Plate Fig. 2), these replacement odontoblasts were derived from the surrounding pulp. The labelling indicates that these cells responded to the wounding and treatment by replicating their DNA and differentiating into functioning odontoblast-like cells.

The existence of adjacent labelled and unlabelled odontoblast-like cells at the exposure site with a similar stage of maturation (Plate Figs 4 and 5) could be explained in at least two ways. The unlabelled cells could represent replacement odontoblasts derived from a population of $\mathrm{G}_{2}$-blocked, predetermined cells that responded to the inducing event by entering M-phase directly and thus not incorporating $\left[{ }^{3} \mathrm{H}\right]-$ thymidine. Such a mechanism has been proposed by Stanley (1962), Takuma and Nagai (1971), and Slavkin (1974). Cotton (1968) and Torneck and Wagner (1980) found no labelled odontoblast-like cells after tooth grinding without exposure and labelling with $\left[{ }^{3} \mathrm{H}\right]$-thymidine. These findings seem to support the $\mathrm{G}_{2}$-blocked replacement cell hypothesis. If such a mechanism were operational, then the most likely source of these cells would be the cell-rich zones of the pulp (Ruch, 1984; Veis, Tsay and Kanwar, 1984). Because the cell-rich zone at the exposure site was destroyed when the pulp was exposed (Plate Fig. 2), the closest available source of undisturbed cell-rich zone remaining would be along the periphery of the exposure. If the unlabelled replacement odontoblast-like cells were from the peripheral cellrich zones, one would expect to find a higher density of this cell type along the edges rather than the centre of the exposure. This assumes that at least some of the recruited replacement cells were not predetermined odontoblasts, as evidenced by the labelled odontoblast-like cells (Plate Figs 4-6). Although we did not look at labelling densities as a function of position along the pulp tissue/capping agent interface, the pattern of labelling among the replacement odontoblasts in zone 1 does not support the notion of recruitment from the periphery (Plate Figs 4 and 5). A more plausible explanation for the presence of the unlabelled replacement odontoblast-like cells is that because the $\left[{ }^{3} \mathrm{H}\right]$-thymidine was available for approximately $1 \mathrm{~h}$ after each injection, these cells had replicated their DNA either before, between, or after the $\left[{ }^{3} \mathrm{H}\right]$-thymidine was available for uptake and thus did not incorporate the marker. This is suggestive of either an asynchronous induction of cells that then undergo final differentiation in a synchronous fashion or a synchronous induction of a non-homogeneous population of cells that reach S-phase at different times but then undergo final differentiation at the same time. The coordination of final differentiation could be related to the establishment of cell-to-cell communications.

Three of our findings suggest that the source of the labelled odontoblast-like replacement cells was the pulp proper, deep to the wound site. These are: (1) the

Plate 1

Fig. 2. Photomicrograph of exposure site (E) and surrounding pulpal tissue (P) at the time of the first $\left[{ }^{3} \mathrm{H}\right]$-thymidine injection. Note the destruction of the odontoblast, cell-free and cell-rich zones as well as deeper pulp tissue. H \& E, original magnification, $125 \times$

Fig. 3. Photomicrograph of zone 1 at day 6 . Note the presence of pulpal tissue directly against the interface with the capping agent $(C)$, the presence of labelled and unlabelled fibroblast-like cells $(F)$ and the absence of odontoblast-like cells. H \& E, original magnification, $625 \times$

Fig. 4. Photomicrograph of zone 1 at day 8 , Note the presence of adjacent labelled and unlabelled odontoblast-like cells $(\mathrm{O})$ associated with new dentine matrix $(\mathrm{M})$ along the interface with the capping agent (C). Toludine blue, original magnification, $625 \times$

Plate 2

Fig. 5. Photomicrograph of zone 1 at day 10 . Note the presence of adjacent labelled and unlabelled odontoblast-like cells $(\mathrm{O})$ associated with new dentine matrix $(M)$ along the interface with the capping agent (C). Toludine blue, original magnification, $625 \times$

Fig. 6. Photomicrograph of zone 1 at day 10 showing two labelled odontoblast-like cell nuclei, one of which is associated with a cell process extending into new dentine matrix $(M)$ along the capping agent interface (C). Toludine blue, original magnification, $1250 \times$ 

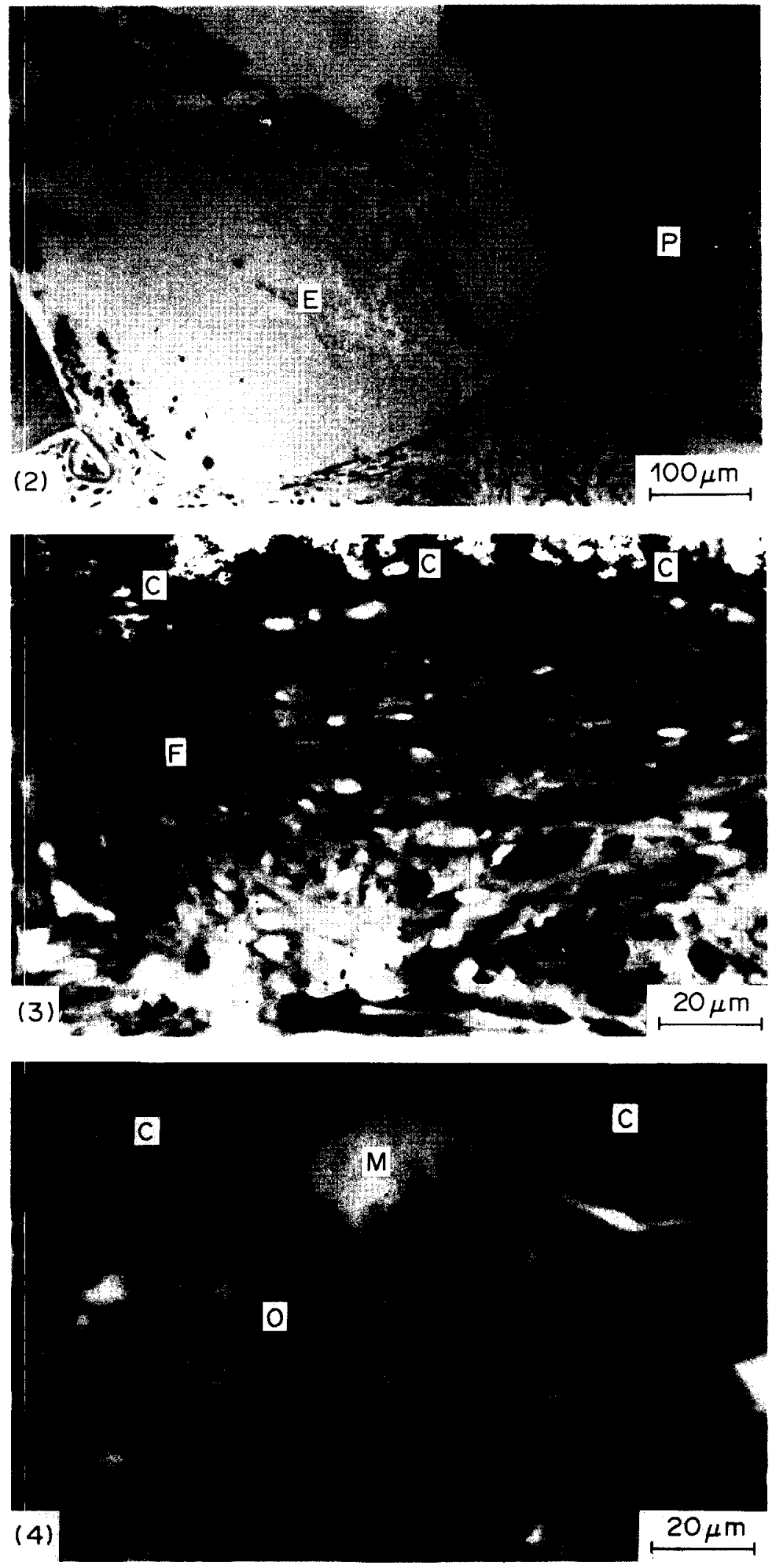

Plate 1 

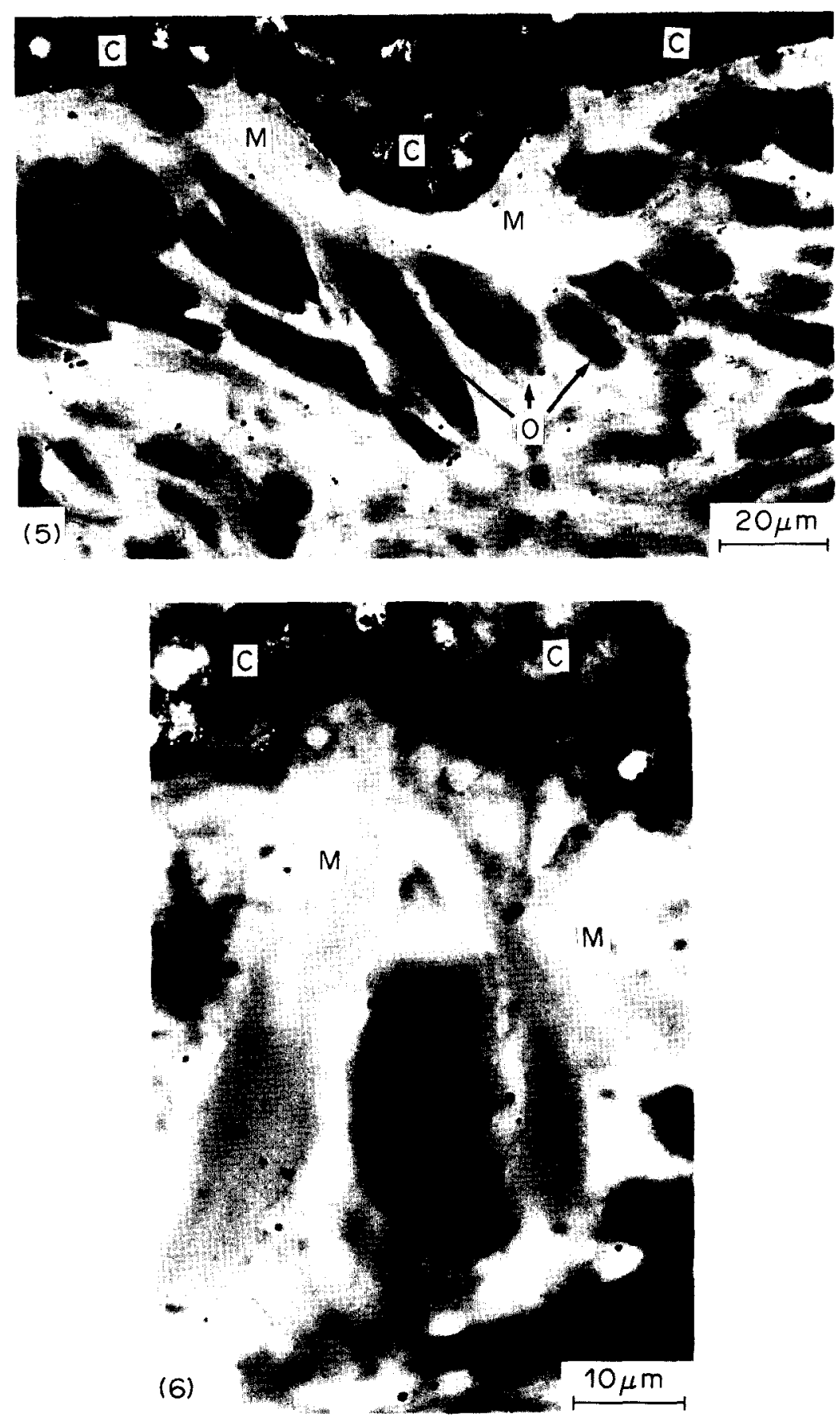

Plate 2 


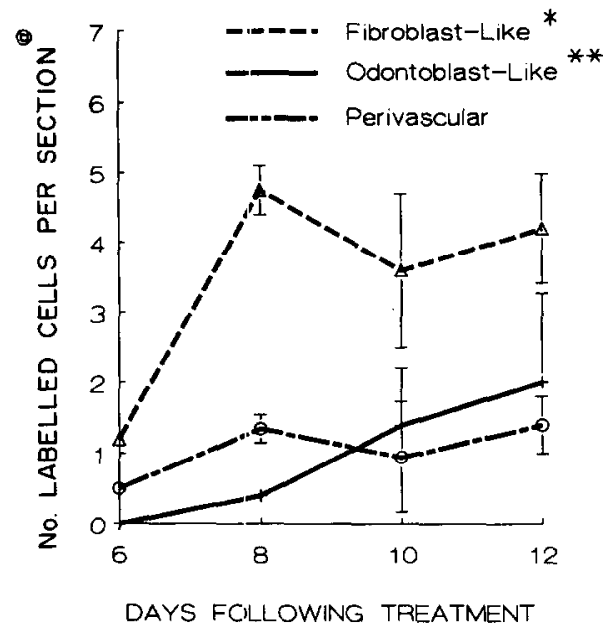

Fig. 7. Number of labelled odontoblast-like, fibroblast-like and perivascular cells in zone 1 for the 4 treatment times. *Labelled fibroblast-like cell counts significantly greater than labelled odontoblast-like and perivascular cell counts. **Significant increase in numbers of odontoblast-like cells over time. @ Total number of labelled cells at day 6 significantly less than at days 8,10 , and 12 , total number of labelled cells at day 8 significantly less than at day 12 and total number of labelled cells at day 10 significantly less than at day 12 .

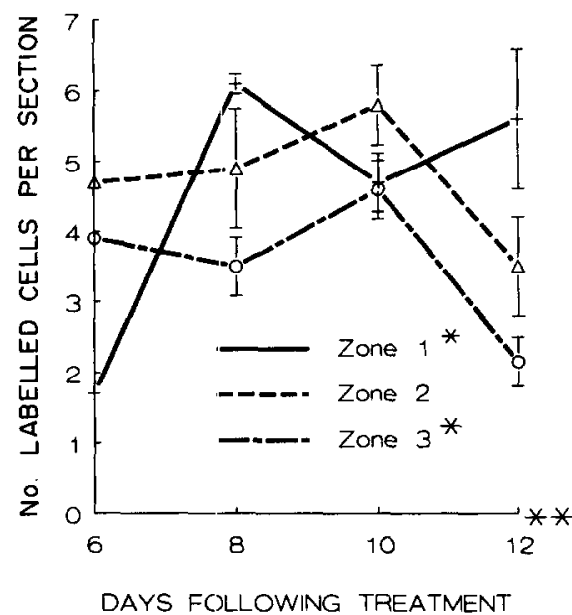

Fig. 8. Number of labelled fibroblast-like plus perivascular cells combined for the 4 treatment times. "Interaction between time periods and zones 1 and 3 ; ** significant difference between zones at day 12 .

significant increase in numbers of labelled odontoblast-like cells in zone 1 over time (Text Fig. 7), suggesting a continual arrival of cells; (2) the combined data for fibroblast-like plus perivascular cells which demonstrate significant shifts from low to high counts of labelled sells in zone 1 and high to low counts in zone 3 (Text Fig. 8), indicating a continuing migration of cells over time from zone 3 to zone 1; and (3) the significantly greater total number of cells in zone 1 than zone 3 at day 12 (Text Fig. 8), demonstrating an influx into and accumulation of cells in zone 1 . These patterns suggest a migration of cells from the deeper pulp (represented by zone 3 ) through an intermediate area (zone 2) into the area directly against the capping agent (zone 1), and support the findings of Sveen and Hawes (1968), Feit et al. (1970), Yamamura et al. (1980) and Yamamura (1985). Tziafas and Kolokuris (1990) demonstrated that cells in the deep pulp can differentiate into odontoblasts when exposed to demineralized dentine surgically implanted in the pulp. Yamamura $e t$ al. (1980) concluded that injury caused by partial pulpotomy capped with pure calcium hydroxide leads either to dedifferentiation of mesenchymal cells in the deep pulp into undifferentiated mesenchymal cells, which then migrate toward the wound, undergo all phases of division and subsequently redifferentiate into new odontoblasts, or to direct induction and differentiation of existing undifferentiated mesenchymal cells. It is not unreasonable to surmise that under our experimental conditions events occurred that were enough to induce migration, DNA replication and differentiation of cells in the deeper pulp into functioning odontoblasts. Identification of the precise inductive event(s) was beyond our scope but is currently being investigated.

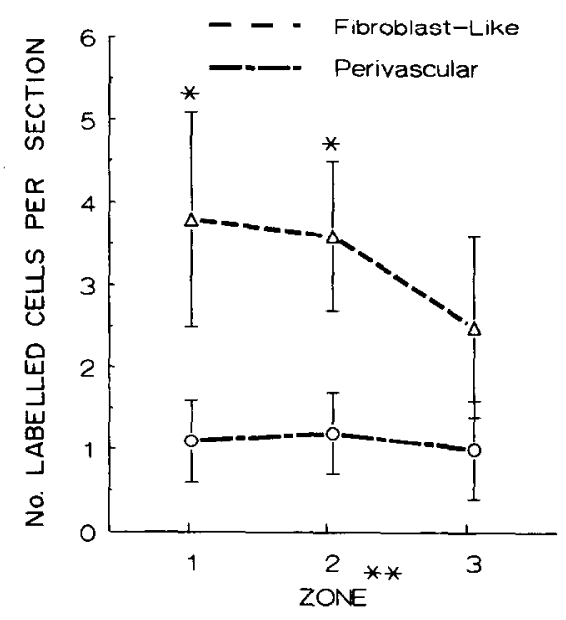

Fig. 9. Total number of labelled fibroblast-like and perivascular cells per section. "Significantly greater number of labelled fibroblast-like cells in zones 1 and $2 .{ }^{* *}$ Number of labelled cells in zones 1 and $2>$ zone 3 .

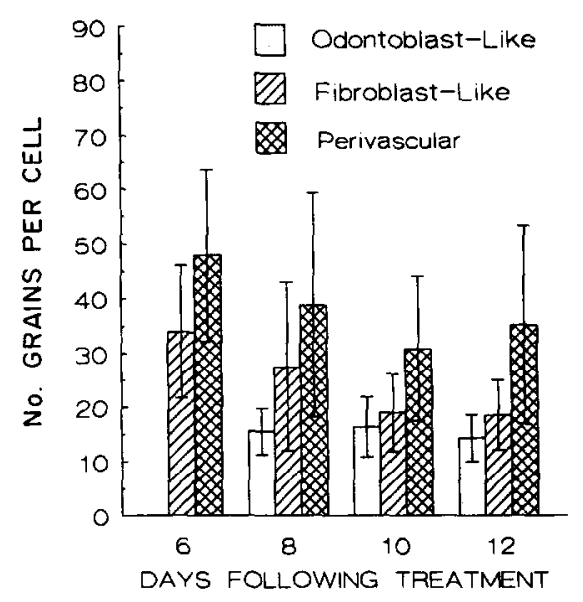

Fig. 10. Average number of grains per cell in zone 1. 


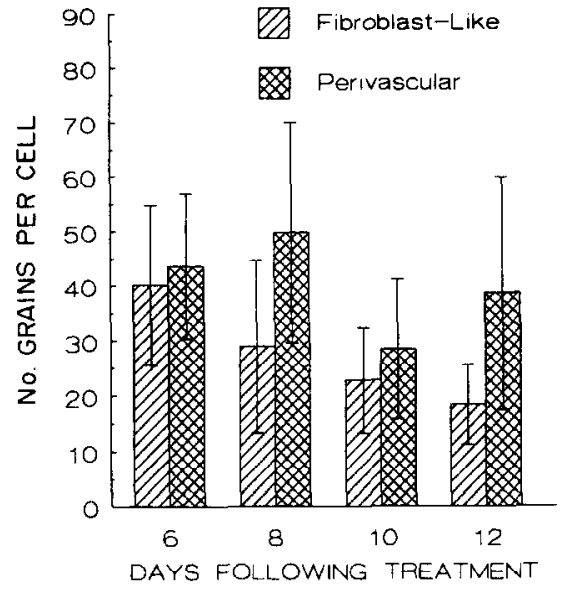

Fig. 11. Average number of grains per cell in zone 2.

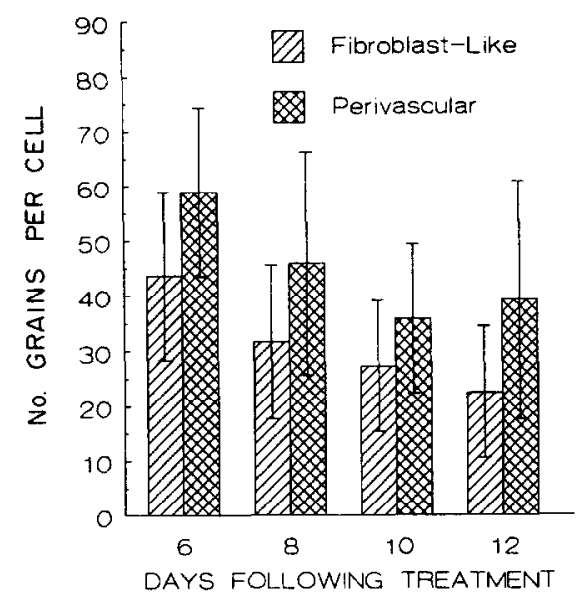

Fig. 12. Average number of grains per cell in zone 3.

Although our findings suport Yamamura's hypothesis of induction and differentiation of pulpal cells, they do not provide direct identification of the specific cell type of the replacement cells. The increase in mean number of labelled odontoblast-like cells per section in zone 1 between Days 8 and 10 with the concurrent and identical decrease in the mean number of labelled fibroblast-like cells per section in zone 1 (Text Fig. 7) suggests a conversion of fibroblast-like cells in zone 1 at 8 days into odontoblast-like cells in zone 1 at 10 days. Such a role for pulpal fibroblasts would not be unreasonable. Indeed, fibroblast-like cells are able to differentiate into either osteoblasts or chondroblasts depending on the nature of the surrounding matrix and the amounts of vascular-derived oxygen (Indue et al., 1981). Given the proper environment, they may also be able to differentiate into replacement odontoblasts, as suggested by Fitzgerald (1979), Yamamura et al. (1980), Yamamura (1985) and Tziafas and Kolokuris (1990). The shift in numbers of fibroblast-like and odontoblast-like cells in zone 1 between Days 8 and 10, was not seen between Days 10 and 12 in zone 1 . This may be due to an influx of labelled fibroblast-like cells from zone 2 during the same time-span.
Further support for the hypothesis that the pulpal fibroblast-like cells are the source of the labelled replacement odontoblasts can be found in the grain counts. The near identical counts for the fibroblastlike and odontoblast-like cells at day 12 zone 1 (Text Fig. 10) suggest that the labelled fibroblast-like cells in zone 1 represent cells that have responded to the trauma and are dedicated to the replacement of the lost odontoblasts. The higher grain counts for fibroblast-like cells in the deeper zones at the later times (Text Figs 11 and 12) suggest that there may be a subpopulation(s) of labelled cells in these zones that are not commmitted to or recruited specifically for odontoblast replacement. A subset of these cells could also represent daughter cells from the time of initial induction that did not migrate to the wound site.

The grain counts also suggest that the labelled odontoblast-like cells underwent at least 2 cell cycles: one represented by the initial uptake of the tritiated thymidine soon after injection and one represented by the 2-fold difference between counts in pulpal cells at day 6 and the counts for the presumably end-stage odontoblast-like cells (Text Figs 10-12). This process of multiple DNA replication before final differentiation is similar to that reported by Ruch (1984) during initial development in a mouse embryonic model. Ruch found that multiple DNA replications, and thus presumably cell divisions, are required before final cytodifferentiation into a functioning primary odontoblast. This final replication was in response to specific and unique alterations within the basement membrane of the embryonic tooth. After this final cell division, one daughter cell remains at the terminal migration point, the basement membrane, while the other migrates to the subjacent pulp. A sequence of cell division similar to that described by Ruch appears to have occurred in the differentiation of at least labelled replacement odontoblasts in the wounded mature teeth used in our study. Our grain counts indicate that these labelled odontoblastlike cells had initially replicated their DNA in the pulp tissue deep to zone 1 before reaching their terminal migration point (the material-pulp interface in zone 1) and then underwent final replication at or near this point. The labelled fibroblast-like cells in zone 1 with grain counts similar to the labelled odontoblast-like cells could represent $\mathrm{G}_{2}$-blocked cells that are a new population of 'predetermined' replacement odontoblast cells; they may thus be similar to the primary odontoblast daughter cells residing in the cell rich zone described by Ruch (1984). However, definitive evidence of the location of the terminal DNA replication as well as of the total number of replications that occur in odontoblast replacement after exposure and pulp capping is still lacking. Studies with more refined techniques for cell kinetics could provide further elucidation. If the terminal DNA replication were to occur at the interface between the pulp tissue and the capping agent, this replication could be triggered by an event or a diffusible substance unique to that interface, as suggested by Ruch (1985).

The definitive role of the labelled fibroblast-like cells remains unclear because of the changing nature of the perivascular cell population. Once a perivascular cell has replicated its DNA and divided, a labelled 
daughter cell that is no longer in direct contact with the outer surface of the blood vessel will appear as a fibroblast-like cell according to the criteria used here. Also, the fibroblast-like cell population most likely contains multiple types including undifferentiated and differentiated lines (Baume, 1980; Yamamura et al., 1980).

Based on our findings and those of others (Fitzgerald, 1979; Yamamura et al., 1980; Ruch, 1984; Tziafas and Kolokuris, 1990), the following hypothesis for the odontoblast cell replacement mechanism would seem reasonable. Odontoblast replacement under our experimental conditions involves multiple DNA replications and migration of pulpal cells to the exposure site from the deeper pulp. These replacement cells appear to be derived from a population of pulpal cells that are deep to the exposure and are fibroblast-like in appearance (although they could contain daughter cells from vascular-related cell groups). After induction, they migrate to the interface between the pulpal tissue and the capping agent where they undergo a final DNA replication and begin functioning as odontoblasts. Although other mechanisms of replacement may also function in conjunction with this, their relative importance appears to be minor.

Acknowledgements - The authors wish to thank John Baker for this technical assistance and Charles J. Kowalski for his invaluable assistance on data analysis. This study was supported by NIH grant NO. RR05321-20. This manuscript is dedicated to Millie Wadsworth.

\section{REFERENCES}

Baume L. J. (1980) The Biology of Pulp and Dentin, Monographs in Oral Science (Edited by H. M. Myers) pp. 123-126, 172-173, Karger, Basel.

Cotton W. R. (1968) Biology of the Dental Pulp Organ (Edited by S. B. Finn) Part 1, pp. 69-90. Univ. of Alabama Press, Birmingham.

Feit J., Metelova M. and Sindelka Z. (1970) Incorporation of ${ }^{3} \mathrm{H}$ thymidine into damaged pulp of rat incisors. J. dent. Res. 49, 783-786.
Fitzgerald M. (1979) Cellular mechanics of dentinal bridge repair using ${ }^{3} \mathrm{H}$-thymidine. $J$. dent. Res. 58, 2198-2206.

Indue T., Saski A., Shimono M. and Yamamura T. (1981) Bone morphogenesis induced by implantation of dentin and cortical bone matrices. Bull. Tokyo Dent. Coll. 22, 213-221.

Ruch J. V. (1984) Dentin and Dentinogenesis, Volume I (Edited by A. Linde) Chap. 3, pp. 47-79. CRC Press, Boca Raton, FL.

Ruch J. V. (1985) Odontoblast differentiation and the formation of the odontoblast layer. J. dent. Res. 64(Spec. Iss.), 489-498.

Slavkin H. C. (1974) Tooth formation: A tool in developmental biology. Oral Sci. Rev. 4, 1-136.

Slavkin H. C., Zeichner-David M. and Siddiqui M. A. Q. (1981) Molecular aspects of tooth morphogenesis and differentiation. Molec. Aspects Med. 4, 125.

Stanley H. R. (1962) The cells of the dental pulp. Oral Surg. $15,849-858$.

Sveen O. B. and Hawes R. R. (1968) Differentiation of new odontoblasts and dentin bridge formation on rat molar teeth after tooth grinding. Archs oral Biol. 13, 1399-1412.

Takuma S. and Nagai N. (1971) Ultrastructure of rat odontoblasts in various stages of their development and maturation. Archs oral Biol. 16, 993-1011.

Torneck C. D. and Wagner D. (1980) The effect of a calcium hydroxide cavity liner on early cell division in the pulp subsequent to cavity preparation and restoration. J. Endocr. 6, 719-723.

Tziafas D. and Kolokuris I. (1990) Inductive influences of demineralized dentin and bone matrix on pulp cells: An approach of secondary dentinogenesis. J. dent. Res. 69, 75-81.

Veis A., Tsay T.-G. and Kanwar Y. (1984) An immunological study of the localization of dentin phosphophoryns in the tooth. INSERM 125, 223-232.

Winer B. J. (1962) Statistical Principles of Experimental Design, Chap. 7, pp. 298-378. McGraw-Hill, New York.

Yamamura T. (1985) Differentiation of pulpal cells and inductive influences of various matrices with reference to pulpal wound healing. J. dent. Res. 64 (Spec. Iss.), 530-540.

Yamamura T., Shimono M., Koike H., Terao M., Tanaka Y., Sakai Y., Inoue T., Yoshiki S., Tachikawa T., Kawahara $H$. and Watanabe O. (1980) Differentiation and induction of undifferentiated mesenchymal cells in tooth and periodontal tissue during wound healing and regeneration. Bull. Tokyo Dent. College 21, 181-222. 